\title{
Verification of the Indicating Measuring Instruments Taking into Account their Instrumental Measurement Uncertainty
}

\author{
Igor Zakharov ${ }^{1}$, Pavel Neyezhmakov², Olesia Botsiura ${ }^{3}$ \\ ${ }^{1}$ Kharkiv National University of Radio Electronics, Department of Metrology and Technical Expertise, Faculty of \\ Infocommunications, University, Nauky Ave., 14, 61166, Kharkiv, Ukraine, newzip@ukr.net \\ ${ }^{2}$ National Scientific Centre “Institute of Metrology”, Myronosytska Str., 42, 61002, Kharkiv, Ukraine, \\ pavel.neyezhmakov@metrology.kharkov.ua \\ ${ }^{3}$ Kharkiv National University of Radio Electronics, Department of Higher Mathematics, Faculty of Information-Analytical \\ Technologies and Management, University, Nauky Ave., 14, 61166, Kharkiv, Ukraine
}

\begin{abstract}
The specific features of the measuring instruments verification based on the results of their calibration are considered. It is noted that, in contrast to the verification procedure used in the legal metrology, the verification procedure for calibrated measuring instruments has to take into account the uncertainty of measurements into account. In this regard, a large number of measuring instruments, considered as those that are in compliance after verification in the legal metrology, turns out to be not in compliance after calibration. In this case, it is necessary to evaluate the probability of compliance of indicating measuring instruments. The procedure of compliance probability determination on the basis of the Monte Carlo method is considered. An example of calibration of a Vernier caliper is given.
\end{abstract}

Keywords: Probability of compliance; uncertainty of measurement; calibration; verification; uncertainty budget; maximum permissible error.

\section{INTRODUCTION}

The items 5.6.2.1.1 and 5.10.4.1.b of ISO 17025:2005 [1] prescribe that calibration certificates for measuring instruments (MIs) shall contain "the measurement results, including the measurement uncertainty and/or a statement of compliance with an identified metrological specification”. From this requirement, it follows that the presence of an indication of conformity of the calibrated MI to the established metrological requirements or separate metrological characteristics is necessary in the calibration certificate. The conformity assessment of the gauge according to the specified requirements is considered in a number of documents [2]-[8]. The main requirement of these documents is the need to take into account the uncertainty of measurement when performing conformity assessment.

\section{SUBJECT \& METHODS}

\subsection{Conformity region and probability of compliance}

Conformity with a specification is proved when the complete measurement result falls within the tolerance region [5]:

$$
L S L \leq y-U \text { or } y+U \leq U S L
$$

where LSL and USL - the lower and upper specification limits of the tolerance region, respectively; $y$ and $U$ - the estimate of measurand and expanded uncertainty, respectively.

These expressions are combined into one, in which the measurement result is within the conformity region [5]:

$$
L S L+U \leq y \leq U S L-U
$$

A measuring instrument may be an indicating measuring instrument (IMI) or a material measure. The measurand of IMI calibration is the systematic error $E_{X}$. In verification of an IMI, the modulus of the specification limits of tolerance region is equal to its maximum permissible error (MPE):

$$
\left|L S L_{\mathrm{IMI}}\right|=U S L_{\mathrm{IMI}}=\mathrm{MPE} .
$$

It should be noted that the main sources of uncertainty of IMI calibration are: instrumental uncertainty of the measurement standard, its instability, changes in its operating conditions, mutual influence of the measurement standard and the IMI to be calibrated; the observed variation in the readings of the calibrated IMI; resolution of a displaying device of IMI. With all uncertainty components taken into account, the extended measurement uncertainty during calibration may be greater than MPE. 
In the example S10 of calibration of a Vernier caliper with a resolution of $0.05 \mathrm{~mm}$, considered in [9], the measurement model is:

$$
E_{X}=l_{i X}-l_{S}+L_{S} \cdot \bar{\alpha} \cdot \Delta t+\delta l_{i X}+\delta l_{M},
$$

where $l_{i X}$ - indication of the caliper; $l_{S}$ - length of the actual gauge block; $L_{S}$ - nominal length of the actual gauge block; $\bar{\alpha}$ - average thermal expansion coefficient of the caliper and the gauge block; $\Delta t$ - difference in temperature between the caliper and the gauge block; $\delta l_{i X}-$ correction for the finite resolution of the caliper; $\delta l_{M}-$ correction for the mechanical effects.

The uncertainty budget is given in Table 1 . There are 2 dominating rectangular contributions in this budget. Therefore, the expanded measurement uncertainty (for trapezoidal distribution law) was:

$$
U=k \cdot u\left(E_{X}\right)=1.83 \cdot 0.0325 \mathrm{~mm} \approx 0.06 \mathrm{~mm} .
$$

The coverage factor 1.83 for trapezoidal distribution is substituted in clause S10.10 [9].

\begin{tabular}{|c|c|c|c|c|c|}
\hline $\begin{array}{c}\text { quantity } \\
X_{i}\end{array}$ & $\begin{array}{c}\text { estimate } \\
X_{i}\end{array}$ & $\begin{array}{c}\text { standard } \\
\text { uncertainty } \\
u\left(x_{i}\right)\end{array}$ & $\begin{array}{l}\text { probability } \\
\text { distribution }\end{array}$ & $\begin{array}{c}\text { sensitivity } \\
\text { coefficient } \\
C_{i}\end{array}$ & $\begin{array}{c}\text { uncertainty } \\
\text { contribution } \\
u_{i}(y)\end{array}$ \\
\hline$l_{i X}$ & $\begin{array}{c}150.10 \\
\mathrm{~mm}\end{array}$ & - & - & - & - \\
\hline$l_{S}$ & $\begin{array}{c}150.00 \\
\mathrm{~mm}\end{array}$ & $0.46 \mu \mathrm{m}$ & rectangular & -1.0 & $-0.46 \mu \mathrm{m}$ \\
\hline$\Delta t$ & 0 & $1.15 \mathrm{~K}$ & rectangular & $1.7 \mu \mathrm{mK}^{-1}$ & $2.0 \mu \mathrm{m}$ \\
\hline$\delta l_{i X}$ & 0 & $14.4 \mu \mathrm{m}$ & rectangular & 1.0 & $14.4 \mu \mathrm{m}$ \\
\hline$\delta l_{M}$ & 0 & $29 \mu \mathrm{m}$ & rectangular & 1.0 & $29 \mu \mathrm{m}$ \\
\hline$E_{X}$ & $0.10 \mathrm{~mm}$ & - & - & - & $32.44 \mu \mathrm{m}$ \\
\hline
\end{tabular}

Table 1. Uncertainty budget of Vernier caliper calibration [9].

So, such a Vernier caliper will be unusable even if its readings do not deviate from the value of the end length gauge, since it is usually equal to its resolution for MPE of the Vernier caliper. If we neglect the unjustifiably high uncertainty associated with the influence of the measuring force (this is quite true for the Vernier calipers with a measuring force control), this will lead to a reduced uncertainty (Table 2.). There is only one dominating rectangular contribution in this budget. Therefore, expanded uncertainty in this case will be:

$$
U=k \cdot u\left(E_{X}\right)=0.95 \sqrt{3} \cdot 0.15=0.0247 \mathrm{~mm} .
$$

The coverage factor $0.95 \sqrt{3}$ for rectangular distribution was taken from the formula (S9.8) [9].

It should be noted that even in this case the condition $U<M P E / 3$, given in [3], is not observed.

\begin{tabular}{|c|c|c|c|c|c|}
\hline $\begin{array}{c}\text { quantity } \\
X_{i}\end{array}$ & $\begin{array}{c}\text { estimate } \\
x_{i}\end{array}$ & $\begin{array}{c}\text { standard } \\
\text { uncertainty } \\
u\left(x_{i}\right)\end{array}$ & $\begin{array}{l}\text { probability } \\
\text { distribution }\end{array}$ & $\begin{array}{c}\text { sensitivity } \\
\text { coefficient } \\
C_{i}\end{array}$ & $\begin{array}{c}\text { uncertainty } \\
\text { contribution } \\
u_{i}(y)\end{array}$ \\
\hline$l_{i X}$ & $\begin{array}{c}150.10 \\
\mathrm{~mm}\end{array}$ & - & - & - & - \\
\hline$l_{S}$ & $\begin{array}{c}150.00 \\
\mathrm{~mm}\end{array}$ & $0.46 \mu \mathrm{m}$ & rectangular & -1.0 & $-0.46 \mu \mathrm{m}$ \\
\hline$\Delta t$ & 0 & $1.15 \mathrm{~K}$ & rectangular & $1.7 \mu \mathrm{mK}^{-1}$ & $2.0 \mu \mathrm{m}$ \\
\hline$\delta l_{i X}$ & 0 & $14.4 \mu \mathrm{m}$ & rectangular & 1.0 & $14.4 \mu \mathrm{m}$ \\
\hline$\delta l_{M}$ & 0 & $3.3 \mu \mathrm{m}$ & triangular & 1.0 & $3.3 \mu \mathrm{m}$ \\
\hline$E_{X}$ & $0.10 \mathrm{~mm}$ & - & - & - & $15 \mu \mathrm{m}$ \\
\hline
\end{tabular}

Table 2. Uncertainty budget of calibration of Vernier caliper with measuring force control.

It should be noted that the expression (1) is true for probability compliance of no more than 0.95 . In general, in the documents [7]-[8] it is proposed to evaluate the probability of compliance of IMIs in the following way:

$$
p_{c}=\Phi_{N}\left(\frac{U S L-|y|}{u}\right)=\Phi_{N}\left(\frac{\mathrm{MPE}-\left|\widehat{E}_{X}\right|}{u}\right)=\Phi_{N}(z),
$$

where $\Phi_{N}(z)$ - standard normal distribution function with variable $z, \hat{E}_{X}, u$ - estimation of IMI's indication error and its standard uncertainty, respectively.

To find $\Phi_{N}(z)$, it is proposed [8] to use the standard normal distribution table (p. 53). However, when calibrating a large number of IMIs, such as Vernier calipers, the distribution function attributed measurand is often trapezoidal or even rectangular. This is due to the fact that the dominant sources of uncertainty of the calibrated IMI are often rectangular distributed corrections, such as the correction of the Vernier caliper resolution [9].

The cumulative distribution function (CDF) of the trapezoidal distribution, which is a convolution of two uniform distributions with the ratio of standard uncertainties $\gamma=u_{2} / u_{1} \leq 1$, has the form:

$$
F_{T}(z)=\left\{\begin{array}{lr}
0, & z<-A ; \\
\frac{\left[z \sqrt{1+\gamma^{2}}+\sqrt{3}(1+\gamma)\right]^{2}}{24 \gamma}, & -A \leq z<-B ; \\
2 \sqrt{1+\gamma^{2}}+\sqrt{3}, & -B \leq z<B ; \\
1-\frac{\left[\sqrt{3}(1+\gamma)-z \sqrt{1+\gamma^{2}}+\right]^{2}}{24 \gamma}, & B \leq z<A ; \\
1, & z \geq A .
\end{array}\right.
$$


where $\mathrm{A}=\sqrt{3}(1+\gamma) / \sqrt{1+\gamma^{2}} ; \mathrm{B}=\sqrt{3}(1-\gamma) / \sqrt{1+\gamma^{2}}$.

For trapezoidal distribution with $\gamma=0.5$ (Table 1.) and considering $z=\left(M P E-\left|\widehat{E}_{X}\right|\right) / u$, we have:

at $\left|\widehat{E}_{X}\right|=0, z=\frac{0.05}{0.0325}=1.538$, that is $p_{c}=0.936<0.95$;

at $\left|\widehat{E}_{X}\right|_{=0.025, z}=\frac{0.025}{0.0325}=0.769$, that is $p_{c}=0.75$;

at $\left|\widehat{E}_{X}\right|=0.05, z=0$, therefore $p_{c}=0.5$.

The rectangular CDF has the form:

$$
F_{R}(z)=\left\{\begin{array}{lr}
0, & z<-\sqrt{3} ; \\
(z+\sqrt{3}) / 2 \sqrt{3}, z \in[-\sqrt{3} ; \sqrt{3}] ; \\
1, & z>\sqrt{3} .
\end{array}\right.
$$

For the rectangular distribution and data of Table 2., we have:

at $\left|\widehat{E}_{X}\right|=0, z=\frac{0.05}{0.015}=3.33>\sqrt{3}$, that is $p_{c}=1$,

at $\left|\widehat{E}_{X}\right|=0.025, z=\frac{0.025}{0.015}=1.67<\sqrt{3}$, that is $p_{c}=0.98$,

at $\left|\widehat{E}_{X}\right|=0.05, z=0$, therefore $p_{c}=0.5$.

Thus, a caliper will be usable with a probability of more than 0.95 if its readings do not deviate from the value of the end length gauge or equal to 0.5 MPE. Practice shows that the number of such calipers is about $60 \%$ of those arrived at the test. Thus, $40 \%$ of the verified Vernier calipers are unusable.

Fig.1. shows the CDF for the uniform, triangular, trapezoidal, and normal distribution laws.

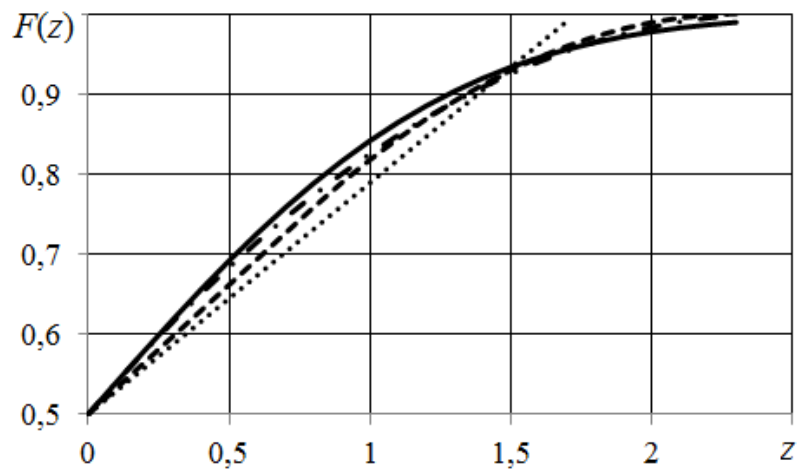

Fig.1. CDF $F(z)$ for uniform ( $\cdots)$, trapezoidal (---) with $\gamma=0.5$, triangular (--- ), and normal (-) distribution laws.

From Fig.1. it is seen that the CDF for the triangular and normal laws practically coincide (with an error of no more than $2 \%$ in probability), therefore, instead of a table with values of the normalized normal distribution given in [8], one can use the dependence for the triangular distribution law:

$$
F_{T}(z)=\left\{\begin{array}{lr}
0, & z<-\sqrt{6} ; \\
(z+\sqrt{6})^{2} / 12, & -\sqrt{6} \leq z<0 ; \\
1-(\sqrt{6}-z)^{2} / 12, & 0 \leq z<\sqrt{6} ; \\
1, & z \geq \sqrt{6} .
\end{array}\right.
$$

The formulas (2) - (4) are obtained for rectangular, triangular and trapezoidal distributions of variable with zero expectations and unit standard deviations. However, these models are only an approximation of the real law of distribution obtained as a result of calibrations. In those cases, we recommend to evaluate the probability of compliance with help of the Monte Carlo method [10].

\subsection{Monte Carlo procedure}

Monte Carlo procedure for construction of distribution function includes the following operations, registered in uncertainty budget (Table 3.):

Table 3. Uncertainty budget.

\begin{tabular}{|c|c|c|c|c|c|}
\hline $\begin{array}{c}\text { input } \\
\text { quantity }\end{array}$ & estimate & $\begin{array}{c}\text { standard } \\
\text { uncertainty }\end{array}$ & $\begin{array}{c}\text { probability } \\
\text { distribution }\end{array}$ & $\begin{array}{c}\text { sensitivity } \\
\text { coefficient }\end{array}$ & $\begin{array}{c}\text { uncertainty } \\
\text { contribution }\end{array}$ \\
\hline$X_{1}$ & $x_{1}$ & $u\left(x_{1}\right)$ & PDF 1 & $c_{1}$ & $u_{1}\left(\widehat{E}_{X}\right)$ \\
\hline$X_{2}$ & $x_{2}$ & $u\left(x_{2}\right)$ & PDF 2 & $c_{2}$ & $u_{2}\left(\widehat{E}_{X}\right)$ \\
\hline$:$ & $:$ & $:$ & $:$ & $:$ & $:$ \\
\hline$X_{N}$ & $x_{N}$ & $u\left(x_{N}\right)$ & PDF $N$ & $c_{N}$ & $u_{N}\left(\widehat{E}_{X}\right)$ \\
\hline $\begin{array}{c}\text { output } \\
\text { quantity }\end{array}$ & estimate & $\begin{array}{c}\text { combined } \\
\text { standard } \\
\text { uncertainty }\end{array}$ & $\begin{array}{c}\text { coverage } \\
\text { probability }\end{array}$ & $\begin{array}{c}\text { coverage } \\
\text { factor }\end{array}$ & $\begin{array}{c}\text { expanded } \\
\text { uncertainty }\end{array}$ \\
\hline$E_{X}$ & $\widehat{E}_{X}$ & $u_{c}\left(\widehat{E}_{X}\right)$ & 0.95 & $k$ & $U$ \\
\hline
\end{tabular}

1. Recording the model equation:

$$
E_{X}=f\left(X_{1}, X_{2}, \ldots, X_{N}\right),
$$

where $X_{1}, X_{2}, \ldots, X_{N}$ - input quantities (first column of the Table 3.).

2. Evaluation of the input quantities as $x_{1}, x_{2}, \ldots, x_{N}$ (second column of the Table 3.).

3. Evaluation of standard uncertainties of the input quantities as $u\left(x_{1}\right), u\left(x_{2}\right), \ldots, u\left(x_{N}\right)$ (third column of the Table 3.).

4. Assigning the probability density functions (PDFs) for input quantities (fourth column of the Table 3.).

5. Selecting the number $M$ of Monte Carlo trials to be made $\left(M \geq 10^{4}\right)$.

6. Generating $M$ trials of measurand for vector, by sampling from the assigned PDFs as realizations of the (set of $N$ ) of the input quantities $X_{i}$.

7. For each such vector, forming the corresponding model value of $E_{X}$, yielding $M$ model values $E_{X i}$. 
8. Calculation of an estimate $\bar{E}_{X}$ of $E_{X}$ by the formula:

$$
\bar{E}_{X}=\frac{1}{M} \sum_{i=1}^{M} E_{X i} .
$$

9. Calculation of unbiased estimate $E_{X i}^{*}$ using the formula:

$$
E_{X i}^{*}=E_{X i}-\bar{E}_{X}
$$

10. Sorting these $M$ model values $E_{X i}^{*}$ into strictly increasing order, using the sorted model values to provide an implementation of the propagation of distributions $G$ [10].

11. Calculating the values of probability

$$
p(i)=100 \cdot i / M, i=1 \ldots M \text {, }
$$

which correspond to the values of $E_{X i}^{*}$.

12. Construction of dependence $p(i)=E_{X i}^{*}$.

13. Finding the probability of compliance $p_{c}$ for the value of

$$
E_{X i}^{*}=M P E-\left|\bar{E}_{X}\right|
$$

Realization of the steps 6-8 of the above described Monte Carlo procedure gives the dependence $p_{c}\left(\mathrm{MPE}-\left|E_{X}\right|\right)$, represented in Fig.2.



Fig.2. Dependence $p_{c}$ of MPE $-\left|E_{X}\right|$.

\section{CONCLUSIONS}

The approaches for compliance probability determination of the IMIs with the specification requirements taking into account the uncertainty of the measurements for the abnormal laws of distribution of their error are presented.

The examples adduced in the article show that for all distribution laws the condition $U<M P E / 3$ given in [3] is not observed.
When carrying out verification of the IMIs applied in the legal metrology, it would also be necessary to take into account the uncertainty of measurements.

\section{REFERENCES}

[1] International Organization for Standardization. (2005). General requirements for the competence of testing and calibration laboratories. ISO/IEC 17025:2005.

[2] EURACHEM/CITAC Working Group. (2012). Quantifying Uncertainty in Analytical Measurement. EURACHEM / CITAC Guide CG4, Third Edition. ISBN 978-0-948926-30-3.

[3] International Organization for Standardization. (2003). Statistical methods - Guidelines for the evaluation of conformity with specified requirements - Part 1: General principles. ISO 10576-1:2003.

[4] EURACHEM/CITAC Working Group. (2007). Use of uncertainty information in compliance assessment. EURACHEM / CITAC Guide, First Edition.

[5] International Organization for Standardization. (2013). Geometrical product specifications (GPS) - Inspection by measurement of workpieces and measuring equipment - Part 1: Decision rules for proving conformity or nonconformity with specifications. ISO 14253-1:2013.

[6] Eurolab. (2008). Determination of conformance with specifications or limit values with particular reference to measurement uncertainties - possible strategies. EUROLAB "Cook Book" - Doc No. 8.0. www.eurolab.org/documents/Cookbook_No_8.pdf.

[7] Joint Committee for Guides in Metrology. (2012). Evaluation of measurement data - The role of measurement uncertainty in conformity assessment. JCGM 106:2012.

[8] International Organization of Legal Metrology. (2017). The role of measurement uncertainty in conformity assessment decisions in legal metrology. OIML G 19, Edition 2017 (E).

[9] EA Laboratory Committee. (2013). Evaluation of the Uncertainty of Measurement In Calibration. EA-4/02 M: 2013.

[10] Joint Committee for Guides in Metrology. (2008). Evaluation of measurement data - Supplement 1 to the "Guide to the expression of uncertainty in measurement" - Propagation of distributions using a Monte Carlo method. JCGM 101:2008.

[11] International Organization for Standardization. (2003). Statistical methods - Guidelines for the evaluation of conformity with specified requirements - Part 1: General principles. ISO 10576-1:2003.

Received September 9, 2017. Accepted November 13, 2017. 